\title{
Opioid Medication Use and Education Following Sports Medicine Procedures: An Evidence-to-Practice Review
}

\author{
Caitlin S. O'Mara, SCAT, ATC; Michael G. Ward, SCAT, ATC; Zachary K. Winkelmann, PhD, SCAT, ATC
}

University of South Carolina, Columbia, SC

\begin{abstract}
According to the Centers for Disease Control (CDC), illicit and prescription drug overdoses are responsible for 128 deaths every day in the United States. In 2018,70\% of all overdose related deaths involved opioids. Efforts to minimize the opioid epidemic focus on community education, research, partnership, and healthcare support. Under the CDC guidelines, current practices include monitoring trends of drug use and drug related deaths, conducting research to recognize areas in need of improvement and to analyze effectiveness of current treatments, partner with community organizations and healthcare systems that deal firsthand with opioid users, and educate the public on drug use, misuse, and overdoses. People are commonly uneducated on the proper use and disposal of their prescription opioids. Consistent and appropriate communication among surgeons and their patients can decrease this risk associated with prescription drugs. The purpose of this evidence-to-practice review was to summarize a systematic review on the current data and findings related to postoperative opioid prescribing and consumption behaviors after a common sports medicine operation. The guiding systematic review explored several ways to reduce the risk of patients developing opioid dependence and abuse due to physicians overprescribing opioids. First, educating each patient about pain management during pre- and postoperative phases, how to store opioids safely, and how to dispose of opioids properly need to be created to help reduce the risk of the patient abusing opioids. Secondly, having the prescribing provider create an extensive history that reveals any red flags for opioid abuse for each patient. Thirdly, the prescribing provider should prescribe the lowest dose and shortest regimen to limit the number of opioids left over. These protocols may help slow the current opioid epidemic.
\end{abstract}

Key Phrases

Drug overdose/drug therapy, program evaluation, opioid-related disorders/drug therapy, naloxone/therapeutic use

\section{Correspondence}

Dr. Zachary Winkelmann, 1300 Wheat Street, Columbia, SC 29208.

E-mail:winkelz@mailbox.sc.edu

Twitter: @zachwinkelmann

\section{Full Citation}

O'Mara CS, Ward MG,Winkelmann ZK. Opioid Medication Use and Education Following Sports Medicine Procedures: An Evidence-to-Practice Review. Clin Pract Athl Train. 2021;4(2): 40-44. https://doi.org/10.31622/2021/0004.2.6.

Submitted: December 15, 2020 Accepted: March 3, 2021.

Copyright (C) by Indiana State University All rights reserved. ISSN Online 2577-8188

\section{ORIGINAL REFERENCE}

Sheth U, Mehta M, Huyke F, Terry MA, Tjong VK. Opioid Use After Common Sports Medicine Procedures: A Systematic Review. Sports Health. $\quad 2020 ; 12(3): 225-233$. https://doi.org/10.1177/1941738120913 293.

\section{SUMMARY}

\section{CLINICAL PROBLEM AND QUESTION}

In n the United States, orthopedic surgeons are responsible for $7.7 \%$ of all prescribed opioids. ${ }^{1}$ When investigating the effects of these practices, two main factors that are studied are mean prescription and consumption values, and appropriate disposal procedures. The guiding systematic review examines these factors following three common procedures: knee, shoulder, and hip arthroscopies. With the increase in the level of skill and competition in athletics, there comes an increased need in medical and surgical knowledge. Studies have shown that there are close to 2 million knee arthroscopic surgeries performed every single year in the United States. ${ }^{2}$ The incidence for the other common injuries (hip and shoulder) are increasing dramatically as well. The drastic increase of these injuries has directly impacted the prescribing patterns of orthopedic surgeons in the United States. Patients and surgeons often assume the use and duration of opioid treatment, that the decision to determine the extent to which the patient needs an opioid prescription, is often overlooked. Regardless of how common the surgery is, or how many times the specific surgeon has performed the surgery, postoperative opioids should be prescribed 
based on the individual patient and with prior research in mind. Supporting research has shown that large percentages of prescribed postoperative opioids are not consumed and/or disposed of properly. ${ }^{3,4}$ In addition to less individualized prescribing patterns, education on proper disposal medication was not always provided. 6 A lack of awareness of proper disposal can often lead to the patient stockpiling their unused medications. The act of collecting pills poses a great risk for future drug abuse or suicidal means. These subsequent behaviors threatened the health and wellbeing of patients, their support systems, and adds to the burden of the current drug epidemic in the United States. Therefore, the purpose of this evidence to practice review was to summarize a systematic review on the current data and findings related to postoperative opioid prescribing and consumption behaviors following three common sports medicine procedures.

\section{SUMMARY OF LITERATURE}

A systematic search was conducted using EMBASE, MEDLINE, and PubMed databases. The keywords searched included arthroscopy, sports medicine, opioids, and other related wording. Inclusion criteria consisted of studies containing patients experiencing an arthroscopic procedure of the knee, hip, or shoulder. Other significant inclusion criteria included evaluation of postoperative opioid prescribing behaviors and evaluated opioid consumption rates. ${ }^{2}$ Studies also needed more than 10 participants and had reports of patient-specific data to be accepted. Quality of the studies was determined using the Methodological Index for Non-Randomized Studies. For these studies; the highest possible score is a 16. All studies had a score of 10 or higher, assigning them high quality. The initial search identified 119 studies. After screening, 8 studies meet the inclusion criteria and were deemed eligible.

Copyright $(\subset)$ by Indiana State University All rights reserved. ISSN Online 2577-8188

\section{SUMMARY OF OUTCOMES}

Five variables were examined in the various studies including opioid prescribing practices, postoperative opioid consumption, duration of opioid use, opioid disposal, and refill rate. During statistical analysis, the guiding review's authors referred to the accepted conversion table from the Centers for Medicare and Medicaid Services. 5 With the oral morphine milligram equivalent conversion factors, the quantity of opioids prescribed, used, and leftover were converted to milligram morphine equivalents (MMEs) for standardized reporting. ${ }^{6}$ In the studies converting opioid prescribing patterns, MMEs are identified for arthroscopic procedures of the shoulder, hip, and knee.

\section{FINDINGS AND CLINICAL IMPLICATIONS}

Among 195 patients ( 3 studies) undergoing the shoulder procedure, there was a mean of 610 MMEs prescribed and 418 MMEs consumed. For 451 patients (4 studies) undergoing the knee procedure, there was a mean of $197 \mathrm{MMEs}$ prescribed and 131 MMEs consumed. For 96 patients (2 studies) undergoing hip procedure, there was a mean of 613 MMEs prescribed and 223 MMEs consumed. 2 To summarize these findings, the percentage of prescribed opioids going unused are: $31 \%$ for shoulder procedures, $34 \%$ for knee procedures, and $64 \%$ for hip procedures. Out of the three surgical procedures, $30 \mathrm{mg}$ tablets of oral codeine were prescribed most frequently. The other most frequently prescribed opioids were a $5 \mathrm{mg}$ tablet of oral hydrocodone and a $5 \mathrm{mg}$ tablet of oxycodone. 6 Two additional studies (277 patients) focused on how frequently post-op patients refill their prescription. The studies concluded that $26 \%$ of these patients asked for a prescription refill following their procedure. This review found overwhelming evidence that opioids are overprescribed for patients that have undergone shoulder, knee, and hip arthroscopic surgery 
despite that evidence has shown that more than one third of opioids prescriptions went used and more than half of the patients do not take the opioids after 3 days following their surgery. Five studies (348 patients) looked at opioid disposal methods or if the patient had any prior education on how to properly dispose of the excess prescription. Out of those 348 patients, only $36 \%$ of patients were given instruction on how to dispose of their medications when they no longer needed them for pain management. ${ }^{1}$

After finding that only $36 \%$ of patients were given instruction on how to properly dispose of their unused medications, there is an increased emphasis on developing strategies to better inform guidelines for both the doctors and the patients. ' An increased focus on better prescribing patterns for doctors along with a more consistent patient education program are two critical components needed to evoke change. One strategy that has been suggested is for surgeons to prescribe the lowest dosage for the shortest duration period.7 Several studies have made dosage recommendations on these three specific procedures as well as alternate strategies for controlling postoperative pain.

The authors of the guiding systematic review discussed the growing risk of the over prescribing patterns of opioids and demonstrated that sports medicine procedures are no exception. The opioid use concerns are not just limited to providers prescribing more than needed, but also to the lack of education given to their patients. Overprescribing and improper disposal methods leads to more opioid medications in the hands of people they don't belong, in turn becoming a contributing factor to the opioid epidemic. This heightens the need for athletic trainers and other healthcare providers to be aware of signs and symptoms of opioid abuse. If the problem can be identified early on, there is more time for an appropriate intervention or referral to be made. This additional time can be the difference

Copyright $(\subset)$ by Indiana State University All rights reserved. ISSN Online 2577-8188 between a healthy post-operative experience and one with long term complications. The guiding systematic review found overwhelming evidence that opioids were overprescribed to patients that have undergone shoulder, knee, and hip arthroscopic surgery. Even with doctors overprescribing opioids, only $36 \%$ of the surgical patients were educated on how to dispose of the medication(s) when they were no longer needed for pain management. ${ }^{1}$ These statistics are not unique to these three surgeries. Over prescription is also seen in foot, ankle, and even upper extremity surgical literature. ${ }^{8}$

\section{CLINICAL BOTTOM LINE}

Due to the ongoing battle with the opioid epidemic, which includes misuse and abuse of opioids, focus has been shifted on creating safer prescription practices. An initial in-depth preoperative medical history can aid the clinician with a more appropriate pain management consideration. Each patient would be assessed for not only past opioid use but other drug dependencies as well.' In addition, each patient would be educated using handouts about the expectations of pain throughout the preoperative and postoperative phases as well as storing and disposing of the medication. According to one systematic review, $5 \%$ of the patients were educated on how to dispose of medication properly, 3 out of 4 patients stored the medication in an unlocked location, and less than $30 \%$ planned to or disposed of any unused opioids. Of that, less than $10 \%$ disposed of the opioids by returning to the pharmacy or flushing down the toilet. ${ }^{8,9}$ The best option for disposing of unused medications is to return them to an official take back location. Take back locations can include pharmacies, police stations, or other approved sites. The Drug Enforcement Agency website allows individuals to enter their zip code and find locations within their area that allow medication take backs. The DEA website also has a "flush list" of what medications are safe to be 
flushed down the toilet. If the medication is not listed on the flush list, the only disposal method is to return to a take back location. Take back locations are the safest option because it minimizes the risk that someone within the home will get a hold of the medication. It also eliminates the risk of a drug contaminating the water if it is flushed down the toilet. If the medication is not on the flush list and there are no take back locations in the area, medications can be disposed of in the trash. The FDA suggests mixing the medication with a substance like cat litter, dirt, or food waste in a sealed plastic bag before placing it in the garbage. ${ }^{10}$ All personal information should be scratched off or removed from the prescription bottles as well before disposal. 10 Another review suggested method to limit over prescription is for providers to administer other analgesics via different intake methods. Several studies within the review have shown decreased opioid use after surgery on patients treated with multiple analgesics during the perioperative phase. 7,11 The guiding systematic review determined there was evidence of a correlation of risk factors that correlates with long-term opioid use such as suicide, other drug dependency, mood disorders, and chronic pain.

Despite not having control of postoperative prescriptions, athletic trainers can be a valuable resource for patients to help them understand their procedure and prescriptions. Athletic trainers can be an advocate for patients to help facilitate proper use and ensure clarity of use through discussions with the prescribing provider and members of the sports medicine team. Ensuring that their patient is aware of proper consumption and disposal is an easy task that can prevent addiction or abuse in the future. The providing prescribers can implement this into practice by establishing communication with a patient throughout the entirety of their surgical process. Early and consistent communication among the orthopedic surgeon, athletic trainer, and the patient can mitigate the risk of opioid addiction.

Copyright $(\subset)$ by Indiana State University All rights reserved. ISSN Online 2577-8188
Athletic trainers are in a unique position to help educate patients on how to properly consume, store, and dispose of their opioids. Athletic trainers can also work with their collaborating physician to develop a plan to avoid postoperative opioid abuse and misuse.

\section{REFERENCES}

1. Sheth U, Mehta M, Huyke F, Terry MA, Tjong VK. Opioid Use After Common Sports Medicine Procedures: A Systematic Review. Sports Health. 2020;12(3):225-233. https://doi.org/10.1177/1941738120913 293.

2. Siemieniuk RAC, Harris IA, Agoritsas $T$, et al. Arthroscopic surgery for degenerative knee arthritis and meniscal tears: a clinical practice guideline. BMJ. 2017;357:j1982. https://doi.org/10.1136/bmi.j1982.

3. Ellis TA, 2nd, Hammoud H, Dela Merced $P$, et al. Multimodal Clinical Pathway With Adductor Canal Block Decreases Hospital Length of Stay, Improves Pain Control, and Reduces Opioid Consumption in Total Knee Arthroplasty Patients: A Retrospective Review. J Arthroplasty. 2018;33(8):2440-2448. https://doi.org/10.1016/i.arth.2018.03.05 $\underline{3}$.

4. Sabatino MJ, Kunkel ST, Ramkumar DB, Keeney BJ, Jevsevar DS. Excess Opioid Medication and Variation in Prescribing Patterns Following Common Orthopaedic Procedures. J Bone Joint Surg Am. 2018;100(3):180-188.

https://doi.org/10.2106/ibjs.17.00672.

5. Centers for Disease Control and Prevention. Analyzing opioid prescription data and oral morphine milligram equivalents (MME). https://www.cdc.gov/drugoverdose/resourc es/data.html. Published October 9, 2020.

6. Centers for Disease Control and Prevention. Understanding the epidemic. https://www.cdc.gov/drugoverdose/epidem ic/index.html. Published 2020, March 19. Accessed.

7. Labrum JT, llyas AM. The opioid epidemic: Postoperative pain management strategies in orthopaedics. JBJS Rev. 2017;5(8):e 14. https://doi.org/10.2106/ibjs.rvw.16.00124. 
8. Merrill HM, Dean DM, Mottla JL, Neufeld SK, Cuttica DJ, Buchanan MM. Opioid Consumption Following Foot and Ankle Surgery. Foot Ankle Int. 2018;39(6):649656.

https://doi.org/10.1177/1071100718757 $\underline{527 .}$.

9. Bicket $M C$, Long JJ, Pronovost PJ, Alexander GC, Wu CL. Prescription Opioid Analgesics Commonly Unused After Surgery: A Systematic Review. JAMA Surg. 2017;152(1 1):1066-1071.

https://doi.org/10.1001/iamasurg.2017.08 31.

10. U.S. Food and Drug Administration. How to safely dispose of unused or expired medicine. https://www.fda.gov/drugs/safe-disposalmedicines/disposal-unused-medicines-whatyou-should-know. Published September 25, 2020.

11. Elkassabany NM, Wang A, Ochroch J, Mattera M, Liu J, Kuntz A. Improved Quality of Recovery from Ambulatory Shoulder Surgery After Implementation of a Multimodal Perioperative Pain Management Protocol. Pain Med. 2019;20(5):1012-1019. https://doi.org/10.1093/pm/pny 152. 